Nicola Scichilone, Alba La Sala, Maria Bellia, Katherine Fallano, Alkis Togias,

Robert H. Brown, Massimo Midiri and Vincenzo Bellia

J Appl Physiol 105:832-838, 2008. First published Jul 10, 2008; doi:10.1152/japplphysiol.01307.2007

You might find this additional information useful...

This article cites 25 articles, 17 of which you can access free at:

http://jap.physiology.org/cgi/content/full/105/3/832\#BIBL

Updated information and services including high-resolution figures, can be found at:

http://jap.physiology.org/cgi/content/full/105/3/832

Additional material and information about Journal of Applied Physiology can be found at: http://www.the-aps.org/publications/jappl

This information is current as of October 17, 2008 . 


\title{
The airway response to deep inspirations decreases with COPD severity and is associated with airway distensibility assessed by computed tomography
}

\author{
Nicola Scichilone, ${ }^{1}$ Alba La Sala, ${ }^{1}$ Maria Bellia, ${ }^{2}$ Katherine Fallano, ${ }^{3}$ Alkis Togias, ${ }^{4}$ Robert H. Brown, ${ }^{3}$ \\ Massimo Midiri, ${ }^{2}$ and Vincenzo Bellia ${ }^{1}$ \\ ${ }^{1}$ Dipartimento di Medicina, Pneumologia, Fisiologia e Nutrizione Umana, Sezione di Pneumologia e Medicina, \\ and ${ }^{2}$ Dipartimento di Biotecnologie e Medicina Legale, Sezione di Scienze Radiologiche, University of Palermo, Palermo, \\ Italy; ${ }^{3}$ Department of Environmental Health Sciences, Johns Hopkins University, Baltimore, Maryland; \\ and ${ }^{4}$ National Institute of Allergy and Infectious Diseases, National Institutes of Health, Bethesda, Maryland
}

Submitted 11 December 2007; accepted in final form 7 July 2008

Scichilone N, La Sala A, Bellia M, Fallano K, Togias A, Brown RH, Midiri M, Bellia V. The airway response to deep inspirations decreases with COPD severity and is associated with airway distensibility assessed by computed tomography. J Appl Physiol 105: 832-838, 2008. First published July 10, 2008; doi:10.1152/japplphysiol.01307.2007.- In patients with mild chronic obstructive pulmonary disease (COPD), the effect of deep inspirations (DIs) to reverse methacholine-induced bronchoconstriction is largely attenuated. In this study, we tested the hypothesis that the effectiveness of DI is reduced with increasing disease severity and that this is associated with a reduction in the ability of DI to distend the airways. Fifteen subjects [Global Initiative for Chronic Obstructive Lung Disease (GOLD) stage I-II: $n=7$; GOLD stage III-IV: $n=8$ ] underwent methacholine bronchoprovocation in the absence of DI, followed by DI. The effectiveness of DI was assessed by their ability to improve inspiratory vital capacity and forced expiratory volume in $1 \mathrm{~s}\left(\mathrm{FEV}_{1}\right)$. To evaluate airway distensibility, two sets of highresolution computed tomography scans [at residual volume (RV) and at total lung capacity] were obtained before the challenge. In addition, mean parenchymal density was calculated on the high-resolution computed tomography scans. We found a strong correlation between the response to DI and baseline $\mathrm{FEV}_{1}$ \%predicted $\left(r^{2}=0.70, P<0.0001\right)$ or baseline $\mathrm{FEV}_{1} /$ forced vital capacity $\left(r^{2}=\right.$ $0.57, P=0.001)$. RV \%predicted and functional residual capacity $\%$ predicted correlated inversely $\left(r^{2}=0.33, P=0.02\right.$ and $r^{2}=0.32$, $P=0.03$, respectively), and parenchymal density at RV correlated directly $\left(r^{2}=0.30, P=0.03\right)$, with the response to DI. Finally, the effect of DI correlated to the change in large airway area from RV to total lung capacity $\left(r^{2}=0.44, P=0.01\right)$. We conclude that loss of the effects of DI is strongly associated with COPD severity and speculate that the reduction in the effectiveness of DI is due to the failure to expand the lungs because of the hyperinflated state and/or the parenchymal damage that prevents distension of the airways with lung inflation.

high-resolution computed tomography; methacholine; airway-parenchyma interdependence; bronchoprovocation; hyperinflation

THE BENEFICIAL EFFECTS OF deep inspiration (DI) in maintaining airway caliber have been documented by ample experimental evidence $(6,7,12,14,23)$. In healthy humans, DIs reverse the effects of a direct spasmogen, such as methacholine (MCh) (6, $7,12,14,20)$, but also protect from these effects when taken before spasmogen exposure $(2,15)$. In asthma, the beneficial effects of DI are reduced or absent $(7,12,14,16,17,23)$.

Address for reprint requests and other correspondence: N. Scichilone, Dipartimento di Medicina, Pneumologia, Fisiologia e Nutrizione Umana, Univ. of Palermo, via Trabucco 180, 90146 Palermo, Italy (e-mail: n.scichilone @libero.it).
Our laboratory has recently demonstrated that the ability of DI to reverse the reduction in lung function by $\mathrm{MCh}$ is also attenuated in individuals with even mild chronic obstructive pulmonary disease (COPD), compared with age-matched healthy subjects (21). In addition, in patients with COPD, the loss of this physiological function correlates with the loss of alveolar attachments on the airways (22). We designed this study to test the hypothesis that the beneficial effect of DI in COPD decreases with increasing disease severity. In conjunction with this hypothesis and with the knowledge that disease severity is also related to the destruction of airway parenchyma (13), we further explored whether the impairment of the effect of DI was associated with inability of a deep inspiratory maneuver to distend the airways and increase their caliber. We measured airway caliber using high-resolution computed tomography (HRCT), as previously demonstrated in healthy individuals and asthmatic subjects (4). Using HRCT density, we also assessed airway parenchymal destruction.

\section{MATERIALS AND METHODS}

Subjects

The study protocol conformed to guidelines issued by the Ethics Committee of the University of Palermo, Palermo, Italy, and all subjects gave written, informed consent before participating in the study. We recruited a total of 15 subjects [age: $69 \pm 9.9$ yr (mean \pm SD); range: 53-90 yr], all but one of whom fulfilled the diagnostic criteria for COPD of the GOLD (Global Initiative for Chronic Obstructive Lung Disease) guidelines (10) (Table 1). One subject did not fulfill the GOLD spirometric criteria for airflow limitation, but he was a chronic smoker with persistent respiratory symptoms, a lung COdiffusing capacity of $58 \%$ predicted, and clear radiological evidence of emphysema on HRCT. Ten subjects were ex-smokers $(47 \pm 13$ pack $\cdot y r$, mean \pm SD), and five were current smokers $(25 \pm 13$ pack $\cdot y r$, mean $\pm \mathrm{SD})$. Subjects were recruited from the Outpatient Clinic for Respiratory Diseases of the University of Palermo. None had suffered from an upper respiratory infection for at least $4 \mathrm{wk}$ before evaluation. At the time of the study, all subjects were clinically stable. Before each study visit, subjects abstained from inhaled, short-acting $\beta$-agonists and/or anticholinergic agents for at least $8 \mathrm{~h}$, or long-acting $\beta$-agonists for at least $24 \mathrm{~h}$. No subject was using leukotriene modifiers or methylxanthines. Subjects were ineligible for the study if they had a history of myocardial infarction, congestive heart failure, cor pulmonale, or arrhythmia. Subjects were also excluded if they had a history of asthma.

The costs of publication of this article were defrayed in part by the payment of page charges. The article must therefore be hereby marked "advertisement" in accordance with 18 U.S.C. Section 1734 solely to indicate this fact. 
Table 1. Demographic and functional characteristics of the subjects

\begin{tabular}{|c|c|c|c|c|c|c|c|c|}
\hline Subjects No. & Age, yr & $\mathrm{FEV}_{1}, \%$ predicted & FVC, \%predicted & $\mathrm{FEV}_{1} / \mathrm{FVC}$ & RV, \%predicted & FRC, \%predicted & TLC, \%predicted & $\% \mathrm{BD}(\mathrm{IVC})$ \\
\hline 2 & 72 & 29 & 62 & 0.31 & 136 & 110 & 122 & -1 \\
\hline 3 & 60 & 41 & 88 & 0.35 & 191 & 185 & 120 & -6 \\
\hline 5 & 78 & 38 & 79 & 0.35 & 152 & 137 & 110 & 0 \\
\hline 6 & 64 & 53 & 68 & 0.59 & 120 & 92 & 86 & 0 \\
\hline 7 & 81 & 42 & 77 & 0.34 & 146 & 137 & 101 & -2 \\
\hline 10 & 69 & 48 & 87 & 0.40 & 103 & 100 & 102 & 0 \\
\hline 11 & 70 & 76 & 88 & 0.63 & 82 & 77 & 84 & 13 \\
\hline $12 \dagger$ & 56 & 80 & 77 & 0.84 & 114 & 87 & 84 & 17 \\
\hline 13 & 72 & 84 & 117 & 0.52 & 137 & 126 & 124 & 13 \\
\hline 14 & 71 & 73 & 99 & 0.60 & 89 & 85 & 87 & 9 \\
\hline 15 & 90 & 67 & 90 & 0.59 & 101 & 95 & 98 & 10 \\
\hline Mean $\pm \mathrm{SE}$ & $69 \pm 2.6$ & $56 \pm 4.6$ & $83 \pm 3.8$ & $0.51 \pm 0.04$ & $121 \pm 7.7$ & $108 \pm 7.8$ & $98 \pm 3.8$ & $4.4 \pm 1.7$ \\
\hline
\end{tabular}

$\mathrm{FEV}_{1}$, forced expiratory volume in $1 \mathrm{~s}$; FVC, forced vital capacity; $\mathrm{FEV}_{1} / \mathrm{FVC}$, ratio of $\mathrm{FEV}_{1}$ to $\mathrm{FVC}$; RV, residual volume; FRC, functional residual capacity; TLC, total lung capacity; \%BD (IVC), percent bronchodilation by deep inspirations based on the inspiratory vital capacity. *For subject 4, who is 61 yr old, the lower limit of normal would be equal to 0.70 . $\dagger$ Subject 12 is a long-term smoker whose symptoms were not explained by a comorbid condition, whose lung CO-diffusing capacity was 59\%, and whose high-resolution computerized tomography showed clear evidence of emphysema.

\section{Study Design and Methods}

Clinical and functional assessments. The study consisted of two phases. In the first phase, clinical and functional assessments were performed. The clinical evaluation included a questionnaire that is a modification of the International Union Against Lung and Tuberculosis Disease bronchial symptom questionnaire (1) and a physical examination. The functional assessment included lung volume determinations by the helium dilution method and evaluation of the ability of DI to reverse the effects of MCh bronchoprovocation on lung function. Total lung capacity (TLC), functional residual capacity (FRC), and residual volume (RV) were determined, and the results were expressed as a percentage of predicted values.

The effectiveness of DIs was determined using MCh bronchoprovocations, as previously reported (21). First, a series of increasing single-dose MCh bronchoprovocations were performed on separate days, at least $24 \mathrm{~h}$ apart, until the targeted reduction in lung function was attained. On each experimental day, baseline lung function confirmed that the constrictive effect of the previous day's MCh had dissipated. The primary outcome in these provocations was the inspiratory vital capacity (IVC), and the targeted reduction of this outcome was $15 \%$. At baseline, three acceptable IVC measurements were obtained, and the highest value was used for analysis. The subject was thereafter instructed to abstain from DIs for $20 \mathrm{~min}$, at which point the MCh dose was delivered. Three minutes later, a single IVC maneuver was performed. If the single IVC maneuver did not satisfy the start-of- and end-of-spirometric criteria for acceptability
(30), the test was discarded and repeated on the following day. In the challenge in which the $15 \%$ or greater reduction in IVC from baseline was reached, the subject was asked to take four consecutive deep breaths. With the addition of the single DI maneuver that was already performed as part of the post-MCh spirometry, a total of five DI maneuvers were performed post-MCh. Immediately after the DIs, another single IVC maneuver was performed (Fig. 1).

To obtain IVC, subjects expired forcefully from end-tidal volume to RV and immediately inhaled to TLC. The spirometric measurement was, therefore, a combined maneuver (a partial followed by a maximal forced expiratory maneuver), which enabled the IVC to be determined as the volume of air inhaled from the RV, which is reached at the end of the partial forced expiration, to the TLC, which is reached with the immediately ensuing DI. IVC was used in these studies because it is dependent on the RV reached at the end of the partial expiratory maneuver and assumes that the effort of the ensuing inspiration to TLC is reproducible. Thus the DI of the IVC maneuver does not influence the outcome. The ability of DI to improve IVC after $\mathrm{MCh}$ was calculated using the ratio of the difference between post-DI IVC and post-MCh IVC over the post-MCh IVC; this was expressed as a percentage and was termed "effect of DI." The formula used was:

$$
\text { Effect of DI }=\frac{\text { post-DI IVC }- \text { post-MCh IVC }}{\text { post-MCh IVC }} \times 100
$$

The combined partial/maximal spirometric maneuver also allows for determination of forced expiratory volume in $1 \mathrm{~s}\left(\mathrm{FEV}_{1}\right)$ and

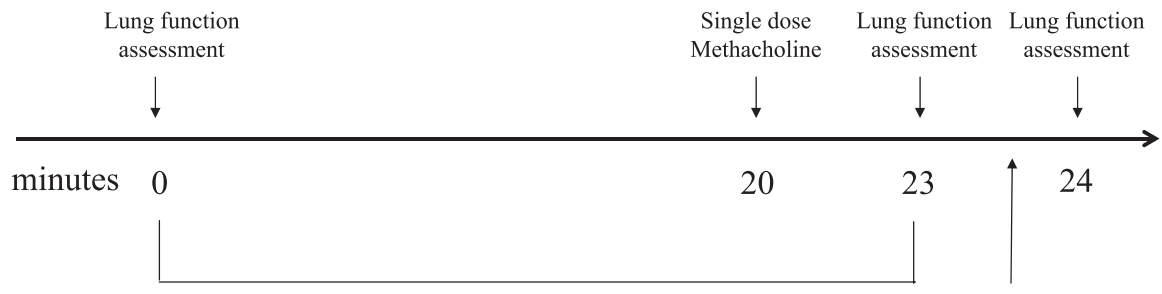

no deep inspiration

4 deep inspirations

Fig. 1. Schematic of the protocol for methacholine (MCh) bronchoprovocations employed to determine the $15 \%$ reduction in inspiratory vital capacity (IVC) and the effect of deep inspiration. Lung function assessment consists of a partial forced expiration from end-tidal volume to residual volume (RV), followed immediately by deep inspiration to total lung capacity (TLC) and by another forced expiration to RV. This combination maneuver enables us to measure the IVC as the difference between the TLC and the preceding RV. Note that a total of five deep inspiration maneuvers were performed after MCh administration and before the last lung function assessment, because one maneuver was already included in the post-MCh lung function assessment. 
forced vital capacity (FVC). These variables were recorded but were only used in secondary analyses due to the fact that their outcome is influenced by the DI of the maximal maneuver; because our study aimed at examining the effects of DI, it was important that the method of measurement of the changes induced by MCh is not itself influenced by DI.

MCh (Lofarma, Milan, Italy) was delivered in tidal inhalations through an ampule-dosimeter (Mefar Elettromedicali, Bovezzo, Italy), which was activated by the inspiratory effort for $0.5 \mathrm{~s}$ at a time. All spirometric measurements were obtained from a computerized, watersealed spirometer (Biomedin, Padua, Italy), which allowed compliance with American Thoracic Society criteria (2a) to be confirmed online.

Airway imaging. On another occasion, we assessed HRCT changes in airway caliber with lung inflation, in the absence of pharmacologically induced airway tone. Each subject underwent HRCT evaluation within 3 days of the functional assessment. HRCT scans were performed by spiral computed tomography (CT) (multidetector 40 channels, Brilliance 40, Philips Medical System, Cleveland, $\mathrm{OH}$ ), with a setting of $120 \mathrm{kVp}, 170 \mathrm{~mA}$, a $2-\mathrm{mm}$ slice thickness, a rotation feed of $2 \mathrm{~cm} / \mathrm{s}$, and a reconstruction interval of $1 \mathrm{~mm}$. Two sets of scans were obtained: one set at RV and the other at TLC. Subjects first exhaled to RV and held their breath; the second set of scans was performed at maximal lung inspiration, at TLC. An initial single-slice reference scan was acquired before each spiral CT scan set, to locate a starting position for the spiral CT. The images were reconstructed as a 16-bit $512 \times 512$ matrix, at a window level of -450 Hounsfield units.

The airway luminal area measurement methodology has been used and validated in previous studies $(4,5)$. Scans through identical airway luminal locations were selected from each of the two sets of scans (RV and TLC) for each subject using adjacent landmarks, such as airway bifurcations or accompanying vessels. The HRCT images were analyzed by using the airway analysis module of the Volumetric Image and Display Analysis software package (Department of Radiology, University of Iowa). The airway luminal area was measured for each airway at RV and TLC. The choice of RV, rather than FRC, as the low volume at which to measure airway areas was made because we were concerned that, due to the expected hyperinflation, the change in airway area from FRC to TLC would be blunted to such an extent that it would not be possible to draw any conclusions from our study. Changes in airway areas were calculated as the ratio of the difference in the airway luminal areas at TLC and at RV divided by the area at RV. For nomenclature of the HRCT measurements of the airways, we arbitrarily divided the airways by their diameter at RV in three groups: small $(<3 \mathrm{~mm}$ in diameter), medium $(3-5 \mathrm{~mm}$ in diameter), and large ( $>5 \mathrm{~mm}$ in diameter) airways. Notably, this airway nomenclature, as defined in the context of the HRCT, is not the same as histologically defined airways, because all of the airways we can measure by HRCT are conducting airways. An example of matched and measured airways on HRCT scans is shown in Fig. 2. A value of airway area change was obtained for small, medium, and large airways. Similarly, airway wall thickness values were obtained for each group of airways. Airway wall thickness was measured by randomly drawing at least three lines through the airway wall. The software program automatically measuring the pixel intensity, and the inflection points of the pixel intensity represent the edges of the inner and outer airway wall perimeters.

In addition, we measured lung parenchymal density on the HRCT scans as an estimate of parenchymal damage. Six regions of interest were placed on each scan where an airway was also measured on the right and left side: one each in the anterior, middle, and posterior part of the parenchyma away from any airways or vessels. An average Hounsfield unit for all of the regions of interest for each subject was calculated.

Data analysis. Data were analyzed by paired $t$-test, as well as by simple linear and stepwise multiple regressions, where appropriate, as

\section{A}

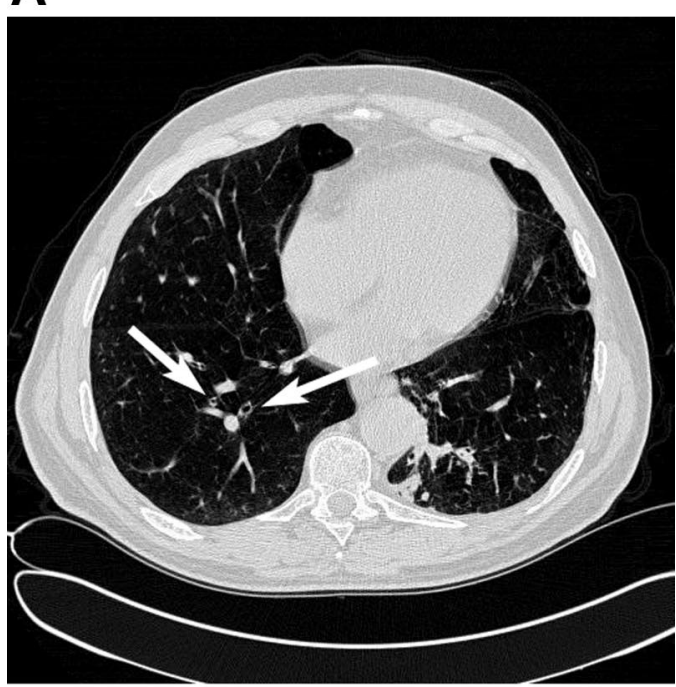

B

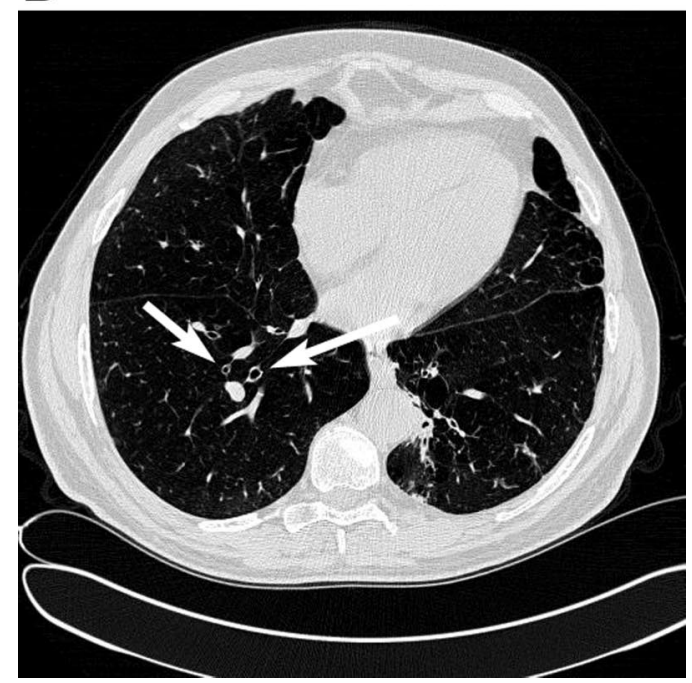

Fig. 2. High-resolution computed tomography (HRCT) images, one obtained at RV $(A)$ and the other at TLC $(B)$, in which examples of matched and measured airways are indicated by the arrows.

indicated below. All data analyses used JMP IN 5.1 software (SAS Institute, Cary, NC). Significance was accepted at a two-tailed level of $P \leq 0.05$. Data are presented in the text as means $\pm \mathrm{SE}$ values.

\section{RESULTS}

The demographics, lung function characteristics, and the DI-induced ability to reverse the effects of MCh on IVC for all subjects who participated in the study are depicted in Table 1. According to the GOLD guidelines for classification of severity (10), six subjects belonged to the mild-to-moderate severity group (stage I-II), and eight subjects were classified as having severe or very severe COPD (stage III-IV). As mentioned before, one individual (subject 12) with no airflow limitation but with a history of smoking habit, dyspnea on exertion in the absence of any comorbid condition, and radiological findings of emphysema was also included in the study.

By definition, $\mathrm{FEV}_{1} \%$ predicted was significantly lower in the individuals who belonged to the stage III-IV group as 


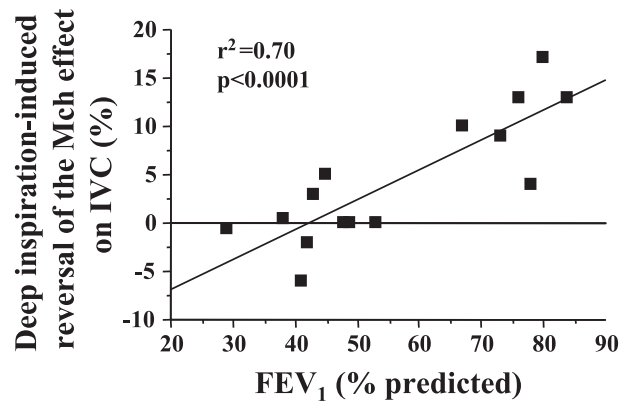

Fig. 3. Correlation between baseline lung function expressed as forced expiratory volume in $1 \mathrm{~s}\left(\mathrm{FEV}_{1}\right) \%$ predicted and the improvement in IVC by deep inspirations after $\mathrm{MCh}$-induced bronchoconstriction.

opposed to stage I-II $(42 \pm 2.2$ vs. $73 \pm 3.9 \% ; P<0.0001$; mean $\pm \mathrm{SE})$. FVC \%predicted was $78 \pm 4.4 \%$ in the stage III-IV and $89 \pm 7.0 \%$ in the stage group I-II $(P=0.17)$, and the $\mathrm{FEV}_{1}$-to-FVC ratio $\left(\mathrm{FEV}_{1} / \mathrm{FVC}\right)$ was $0.39 \pm 0.03$ and $0.64 \pm 0.04$ in stages III-IV and I-II, respectively $(P<$ 0.0001). Also, the RV \%predicted and the FRC \%predicted were higher in the subjects with more severe disease (for RV \%predicted: $135 \pm 11$ vs. $105 \pm 7.5 \%, P=0.04$; for FRC \%predicted: $124 \pm 11$ vs. $91 \pm 6.7 \%, P=0.02$; stage III-IV vs. stage I-II, means \pm SE).

We assessed the effect of DI in reversing the induced bronchoconstriction. The overall reduction in IVC from baseline in the absence of DIs in the single-dose MCh bronchoprovocation protocol was $25 \pm 3.8 \%$. The corresponding reduction in $\mathrm{FEV}_{1}$ from baseline was $15 \pm 3.4 \%$. In one subject with very severe disease and two subjects with severe disease, the bronchoprovocation was stopped, for safety reasons, when subjects reached a reduction in IVC equal to 10, 11, and $13 \%$, respectively, but the protocol was carried on with the DI component. As we have previously demonstrated, in the subject group as a whole, the effect of DI was very low, $4.4 \pm$ $1.7 \%$. Notably, three subjects had negative indexes, as the DIs after MCh caused further reductions in IVC.

The impairment of the effectiveness of DIs was associated with the severity of COPD. The effectiveness of DIs, based on IVC, correlated with baseline $\mathrm{FEV}_{1} \%$ predicted $\left(r^{2}=0.70\right.$, $P<0.0001)$ (Fig. 3) and $\mathrm{FEV}_{1} / \mathrm{FVC}\left(r^{2}=0.57, P=0.001\right)$. The relationship between the effect of DI and FVC \% predicted did not reach statistical significance $\left(r^{2}=0.20, P=0.11\right)$. In addition, a significant inverse relationship was found between indexes of hyperinflation, such as RV \%predicted and FRC $\%$ predicted, and the effect of DI $\left(r^{2}=0.33, P=0.02\right.$ and $r^{2}=0.32, P=0.03$, respectively). We also calculated the effect of DIs on the basis of the $\mathrm{FEV}_{1}$ in addition to the IVC. In this analysis, we only utilized the 10 subjects who had more than $10 \%$ MCh-induced reduction in $\mathrm{FEV}_{1}$ from baseline, in the absence of DIs, because our laboratory has previously shown that, if the reduction in $\mathrm{FEV}_{1}$ is small, the effect of DI becomes difficult to detect (15). The $\mathrm{FEV}_{1}$-based effect of DI in these subjects was $5.7 \pm 1.8 \%$. Contrary to our finding with the IVC-based effect of DI, the $\mathrm{FEV}_{1}$-based effect did not correlate with the severity of baseline airway obstruction expressed as $\mathrm{FEV}_{1} \%$ predicted $\left(r^{2}=0.06, P=0.52\right)$.

The HRCT-determined mean parenchymal density, measured at RV, correlated with the effectiveness of DIs $\left(r^{2}=\right.$ 0.30, $P=0.03$ ) (Fig. 4A). The lower the mean parenchymal density (i.e., lung hyperinflation or lung destruction), the less effective the DIs. In addition, the change (reduction) in parenchymal density from RV to TLC was positively correlated with the effect of DI $\left(r^{2}=0.42, P=0.01\right)$ (Fig. $\left.4 B\right)$. In other words, the greater the ability to inflate the lungs with DI, the more effective the DI-induced reversal of the lung function impairment caused by MCh. Interestingly, the three individuals in whom DIs led to worsening of IVC after MCh (marked with triangles in Fig. 4) were among the subjects with the lowest parenchymal density at RV and those with smallest reduction in parenchymal density from RV to TLC.

A total of 233 airways were matched and measured on the HRCT scans (range: $2.0-17.8 \mathrm{~mm}$ in diameter at RV). For all airways, lung inflation from RV to TLC increased the average airway area by $49 \pm 4.3 \%$ (mean \pm SE). As expected, the relative increase in airway area was higher for the small airways $(74 \pm 7.7 \%)$ as opposed to the medium $(52 \pm 6 \%)$ and large $(17 \pm 8 \%)$ airways $(P<0.0001)$.

Overall, airway area changes from RV to TLC did not correlate with $\mathrm{FEV}_{1} \%$ predicted $\left(r^{2}=0.02, P=0.57\right)$ nor with $\mathrm{FEV}_{1} / \mathrm{FVC}\left(r^{2}=0.01, P=0.72\right)$. However, when airway area changes were analyzed separately according to their baseline caliber, changes in the large airways, but not in the medium and small airways, were significantly associated with $\mathrm{FEV}_{1}$ $\%$ predicted $\left(r^{2}=0.58, P=0.002\right)$ and $\mathrm{FEV}_{1} / \mathrm{FVC}\left(r^{2}=0.34\right.$, $P=0.03$ ) (Fig. 5, $A$ and $B$, respectively). In addition, a relationship that did not reach statistical significance was observed between the RV-to-TLC-induced changes in the area

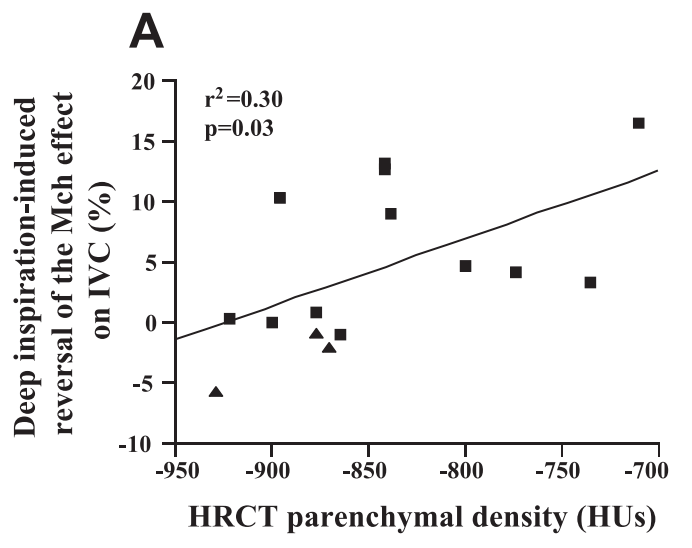

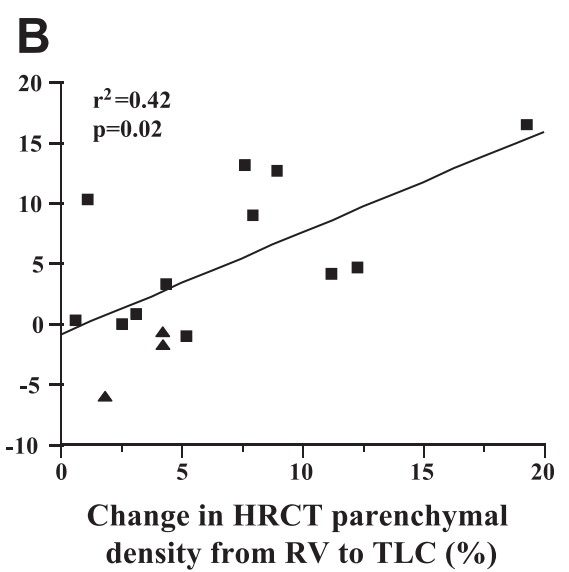

Fig. 4. A: correlation between the improvement in IVC by deep inspirations after MChinduced bronchoconstriction and the lung parenchymal density, as assessed by HRCT. $B$ : correlation between the improvement in IVC by deep inspirations after MCh-induced bronchoconstriction and the changes in the parenchymal density. Parenchymal density was measured in the HRCT scans performed at RV. The triangles represent the three subjects in whom deep inspirations led to reduction in IVC. HU, Hounsfield unit. 
Fig. 5. Correlation between baseline lung function, expressed as $\mathrm{FEV}_{1} \%$ predicted $(A)$, and correlation between baseline lung function, expressed as $\mathrm{FEV}_{1}$-to-forced vital capacity (FVC) ratio $(B)$ and the changes in the large airways areas induced by a single deep inspiratory maneuver.

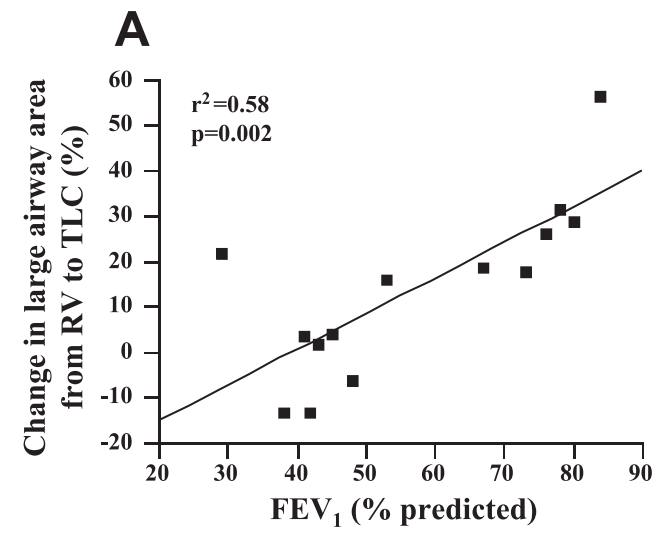

of the large airways and changes in parenchymal density $\left(r^{2}=\right.$ $0.23, P=0.08$ ).

Overall, the change in airway areas by DI was not associated with the beneficial effect of DI against MCh $\left(r^{2}=0.03, P=\right.$ 0.54). Again, however, large airway area changes were significantly associated with the effects of DI $\left(r^{2}=0.44, P=0.01\right)$ (Fig. 6), whereas changes in medium and small airway areas were not $\left(r^{2}=0.008 P=0.75\right.$ and $r^{2}=0.02, P=0.63$, respectively). Airway wall thickness was not associated with the effect of DIs, either overall or for any airway caliber group.

\section{DISCUSSION}

We have shown that the effect of a DI is strongly related to the severity of COPD, based on various aspects of baseline lung function and on imaging abnormalities, such as an HRCTbased index of hyperinflation/parenchymal destruction. In addition, by applying HRCT methodology to assess changes in airway lumen following a deep inspiratory maneuver, we have observed that the ability of DI to reverse the effects of MCh correlates with the ability of DI to distend large airways. These novel observations, which extend our laboratory's previous finding that the effect of DI is depressed in COPD (21), are consistent with the concept that the impairment of DI-induced airway distension and reversal of airway tone may be an important factor contributing to the severity of COPD.

The importance of DI is well appreciated in physiological terms, but very few studies have examined it in the clinical context. A fundamental hypothesis is that DI exerts its effects

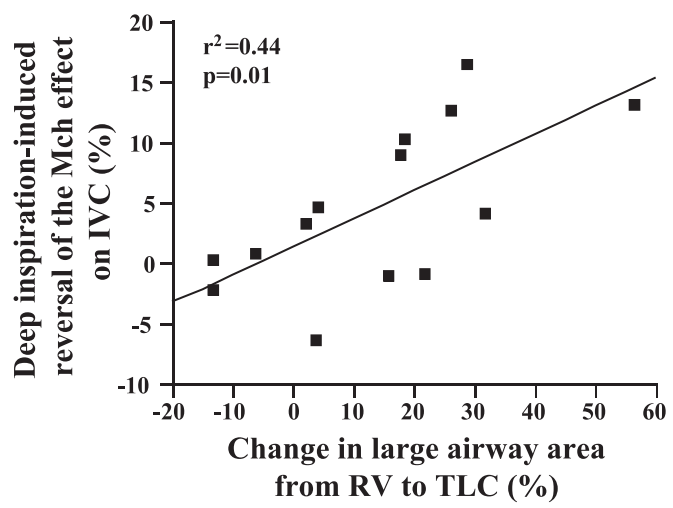

Fig. 6. Correlation between the improvement in IVC by deep inspirations after $\mathrm{MCh}$-induced bronchoconstriction and the changes in the large airways areas induced by a single deep inspiratory maneuver as measured by HRCT. by stretching airway walls. The mechanisms through which airway stretch may result in the beneficial effects of DI are not known, but may include the generation of neural reflexes, release of mediators altering the ASM contractility or, simply, ASM stretch, which can directly influence the functional state of the contractile apparatus $(9,11,19)$. For stretch to be exerted on the airway wall, the anatomic and functional interdependence between the lung parenchyma and the airways needs to be intact. In COPD, alveolar attachments on airway walls are progressively destroyed, and this is expected to impact on the ability of lung inflation by DI to stretch the airways. In fact, we have previously reported a correlation between loss of alveolar attachments and reduction in the beneficial effects of DI (22). In individuals with more severe emphysema, a DI could even lead to bronchoconstriction, as we have previously described in COPD subjects (21). Indeed, when the airways are decoupled from the surrounding parenchyma, the airway response to a DI may convert from dilatory to constrictive (18), most probably because of the reduced load that is offered to the airway smooth muscle by damaged lung parenchyma.

The present study shows that, in COPD, the ability of DIs to reverse the effect of MCh decreased significantly with decreased lung function. The most striking relationship we observed was that baseline $\mathrm{FEV}_{1}$ \%predicted accounted for $77 \%$ of the variance of the DI effect. In COPD, reduced baseline $\mathrm{FEV}_{1}$ results from loss of elastic recoil, hyperinflation, and increased airway tone. Airway wall thickening may also play a small role (25). As will be discussed below, the same factors are probably responsible for the reduction of the effectiveness of DIs. Furthermore, it is possible that the reduced effectiveness of a DI per se can lead to increased airway tone. Given the common upstream pathophysiological mechanisms and the direct interaction, it was not surprising why such a strong association between $\mathrm{FEV}_{1}$ \%predicted and the DI effect was observed.

Baseline functional and radiological descriptors of air trapping and lung hyperinflation, such as RV \%predicted and FRC \%predicted, as well as an HRCT-based index of parenchymal density, also correlated with the effect of DI, with greater hyperinflation and reduced density predicting a reduction in the DI effect. In the hyperinflated state, the ability to generate high transpulmonary pressure is severely decreased, leading to diminished radial traction and distension of the airways; this can reduce the DI effect. In support of this concept, Brown and Mitzner (5) and Salerno et al. (20) have shown that the 
magnitude of lung inflation during a DI has a major effect on subsequent airway size. This is also confirmed by in vitro studies $(9,11)$. In particular, Salerno and colleagues demonstrated that the attenuation of induced bronchoconstriction by applied stretch is linearly associated with the stretch's amplitude. The authors also confirmed that the beneficial effect of lung inflation is more evident on RV than on partial flow, suggesting that small airways are more susceptible to stretchinduced changes. It is, therefore, not surprising that failure to expand the lungs due to the hyperinflated condition would be one of the major determinants of the loss of the beneficial effect of DI.

A major finding of this study was that the capacity of DI to reverse the effects of $\mathrm{MCh}$ was related to its ability to distend the airways. This finding advances our understanding of the function of DI, as it offers strong support to the hypothesis that the ability of DI to reverse induced tone depends on the stretch exerted on airway walls. The relationship between airway stretch and the functional effect of DI may be complicated, and this correlation, albeit quite impressive, does not unveil potential important intermediary interactions. As discussed earlier, airway stretch may activate various mechanisms, including alterations in ASM contractility. In this respect, the fact that five deep breaths were used to reach an optimal functional effect (15), whereas airway distensibility was assessed with a single deep inspiratory maneuver (scanning at RV and TLC), may further confound this relationship. In COPD, reduced ability of DIs to distend the airways probably reflects the loss of airway-parenchyma interdependence that results from parenchymal damage (22). However, as discussed above, parenchymal damage also leads to hyperinflation, which can further interfere with the ability of DI to distend the airways. Another factor that interferes with airway distensibility is smooth muscle tone. Previous work in humans (4) and animals (3) has demonstrated that increased airway smooth muscle tone reduces the ability to pull open the airways with lung inflation. It is important to note that, in our study, airway distensibility by a DI was assessed at baseline and not after the administration of MCh. It is possible that, under the presence of induced tone, the correlation between the effects of DI on lung function and on airway area might have been different; we expect it to have been stronger. However, from a safety and technical perspective, it would have been quite challenging to obtain HRCT measurements at RV and TLC in subjects with severe COPD following the administration of a spasmogen.

Interestingly, we found that the effect of a DI to reverse $\mathrm{MCh}$-induced bronchoconstriction was associated with baseline distensibility by DI of large-, but not small- or mediumsized, airways. This finding seems somewhat counterintuitive because, if the DI effect depends on airway-parenchyma interdependence, it should primarily influence the smaller airways on which alveolar septa exert strong traction. One could attribute this apparent discrepancy between fact and expectation to various technical issues, such as the above-described difference in the circumstances in which the HRCT and the functional assessment of the effect of DI took place or to the fact that, in the HRCT protocol, only a single DI takes place vs. five in the post-MCh assessment of the DI effect. Another explanation can be that, in COPD, the component of airwayparenchyma interdependence attributable to the small- and medium-sized airways is already reduced substantially as a result of parenchymal damage, so that its influence on airway distention, as assessed by HRCT imaging, is negligible. Also, it is important to note that airways smaller than 2-mm diameter at RV cannot be assessed by HRCT, but these airways are probably of importance for the outcome in question. Alternatively, the airway-parenchymal interaction involving the large airways may not be so surprising, since this is the "choke point" in terms of the smallest total cross-sectional airway area throughout the entire airway tree and thus the location of the highest airway resistance to flow in the airway tree (8). As a result, a distending effect by DI on large airways should have great impact on relieving airflow obstruction induced by tone.

We measured the ability of DI to reverse the effects of MCh using two outcomes: the primary being based on IVC and the secondary on $\mathrm{FEV}_{1}$. Although methodological reasons offer a compelling argument for the use of IVC in this line of testing (see MATERIALS AND METHODS), the fact that IVC and $\mathrm{FEV}_{1}$ reflect different aspects of lung function (volume vs. airflow) justifies the collection of data from both outcomes. In this study, however, the index of the effect of DI that was based on $\mathrm{FEV}_{1}$, unlike that based on IVC, did not correlate with the severity of baseline airway obstruction expressed as $\mathrm{FEV}_{1}$ $\%$ predicted $\left(r^{2}=0.06, P=0.52\right)$. Regardless of the reason, this finding attests to a higher discriminatory ability of the IVC-based index in assessing disease severity. In this context, it should be mentioned that the increase in RV is considered the major correlate to dyspnea (24), and that recovery from an exacerbation of COPD is characterized by an increase in inspiratory capacity more than by improvements in expiratory airflows (24).

In conclusion, this work makes two significant contributions to our understanding of the disease process in COPD, as well as our understanding of the mechanism of action of DIs. First, our work demonstrates that, in COPD, the ability to reverse the effects of MCh by DI is reduced in relation to the severity of disease. This supports the notion that the loss of the effect of DI may influence the clinical presentation of COPD. Second, this work advances our understanding of the reduction in the ability of DI to reverse airway tone as it demonstrates a strong relationship between this reduction and large airway distensibility by DI.

\section{GRANTS}

The study was funded by the University of Palermo, Palermo, Italy.

\section{REFERENCES}

1. Abramson MJ, Hensley MJ, Saunders NA, Wlodarczyk JH. Evaluation of a new asthma questionnaire. J Asthma 28: 129-139, 1991.

2. Allen ND, Davis BE, Hurst TS, Cockcroft DW. Difference between dosimeter and tidal breathing methacholine challenge: contributions of dose and deep inspiration bronchoprotection. Chest 128: 4018-4023, 2005

2a.[Anon]. Standardization of Spirometry, 1994 Update. American Thoracic Society. Am J Respir Crit Care Med 152: 1107-1136, 1995.

3. Brown RH, Mitzner W, Wagner EM. Interaction between airway edema and lung inflation on responsiveness of individual airways in vivo. $J$ Appl Physiol 83: 366-370, 1997.

4. Brown RH, Scichilone N, Mudge B, Diemer FB, Permutt S, Togias A. High-resolution computed tomographic evaluation of airway distensibility and the effects of lung inflation on airway caliber in healthy subjects and individuals with asthma. Am J Respir Crit Care Med 163: 994-1001, 2001.

5. Brown RH, Mitzner W. Airway response to deep inspiration: role of inflation pressure. J Appl Physiol 91: 2574-2578, 2001 
6. Brusasco V, Crimi E, Barisione G, Spanevello A, Rodarte JR, Pellegrino R. Airway responsiveness to methacholine: effects of deep inhalations and airway inflammation. J Appl Physiol 87: 567-573, 1999.

7. Crimi E, Pellegrino R, Milanese M, Brusasco V. Deep breaths, methacholine, and airway narrowing in healthy and mild asthmatic subjects. J Appl Physiol 93: 1384-1390, 2002.

8. Dawson SV, Elliott EA. Wave-speed limitation on expiratory flow-a unifying concept. J Appl Physiol 43: 498-515, 1977.

9. Fredberg JJ, Inouye D, Miller B, Nathan M, Jafari S, Raboudi SH, Butler JP, Shore SA. Airway smooth muscle, tidal stretches, and dynamically determined contractile states. Am J Respir Crit Care Med 156: 1752-1759, 1977.

10. Global Initiative for Chronic Obstructive Lung Disease. Global Strategy for the Diagnosis, Management, and Prevention of Chronic Obstructive Pulmonary Disease (Online). http://www.goldcopd.com/Guidelineitem. asp? $11=2 \& 12=1 \&$ intId $=1815$ [2006].

11. Gunst SJ, Wu MF. Selected contribution: plasticity of airway smooth muscle stiffness and extensibility: role of length-adaptive mechanisms. J Appl Physiol 90: 741-749, 2001.

12. Jackson AC, Murphy MM, Rassulo J, Celli BR, Ingram RH Jr. Deep breath reversal and exponential return of methacholine-induced obstruction in asthmatic and nonasthmatic subjects. J Appl Physiol 96: 137-142, 2004.

13. Jeffery PK. Remodeling in asthma and chronic obstructive lung disease. Am J Respir Crit Care Med 164: S28-S38, 2001.

14. Jensen A, Atileh H, Suki B, Ingenito EP, Lutchen KR. Selected contribution: airway caliber in healthy and asthmatic subjects: effects of bronchial challenge and deep inspirations. J Appl Physiol 91: 506-515, 2001.

15. Kapsali T, Permutt S, Laube B, Scichilone N, Togias A. Potent bronchoprotective effect of deep inspiration and its absence in asthma. J Appl Physiol 89: 711-720, 2000.
16. King GG, Moore BJ, Seow CY, Pare PD. Time course of increased airway narrowing caused by inhibition of deep inspiration during methacholine challenge. Am J Respir Crit Care Med 160: 454-457, 1999.

17. Lutchen KR, Jensen A, Atileh H, Kaczka DW, Israel E, Suki B, Ingenito EP. Airway constriction pattern is a central component of asthma severity: the role of deep inspirations. Am J Respir Crit Care Med 164: 207-215, 2001.

18. Mitzner W, Brown R. Potential mechanism of hyperresponsive airways. Am J Respir Crit Care Med 161: 1619-1623, 2000.

19. Nijkamp FP, Folkerts G. Nitric oxide and bronchial reactivity. Clin Exp Allergy 24: 905-914, 1994.

20. Salerno FG, Pellegrino R, Trocchio G, Spanevello A, Brusasco V, Crimi E. Attenuation of induced bronchoconstriction in healthy subjects: effects of breathing depth. J Appl Physiol 98: 817-821, 2005.

21. Scichilone N, Marchese R, Catalano F, Vignola AM, Togias A, Bellia V. Bronchodilatory effect of deep inspiration is absent in subjects with mild COPD. Chest 125: 2029-2035, 2004.

22. Scichilone N, Bruno A, Marchese R, Vignola AM, Togias A, Bellia V. Association between reduced bronchodilatory effect of deep inspiration and loss of alveolar attachments. Respir Res 6: 55, 2005.

23. Skloot G, Permutt S, Togias A. Airway hyperresponsiveness in asthma: a problem of limited smooth muscle relaxation with inspiration. J Clin Invest 96: 2393-2403, 1995.

24. Stevenson NJ, Walker PP, Costello RW, Calverley PM. Lung mechanics and dyspnea during exacerbations of chronic obstructive pulmonary disease. Am J Respir Crit Care Med 172: 1510-1516, 2005.

25. Tiddens HA, Bogaard JM, de Jongste JC, Hop WC, Coxson HO, Pare PD. Physiological and morphological determinants of maximal expiratory flow in chronic obstructive lung disease. Eur Respir J 9: 1785-1794, 1996.

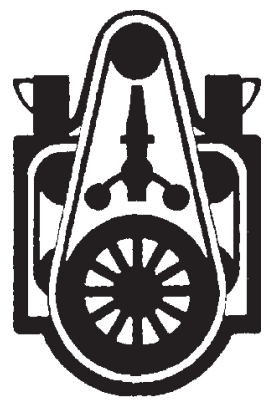

